\title{
Diagnosis of oligometastasis
}

\author{
Mayako Terao, Naoki Niikura \\ Department of Endocrine and Breast Surgery, Tokai University School of Medicine, Isehara, Kanagawa 259-1193, Japan \\ Contributions: (I) Conseption and design: All authors; (II) Administrative support: N Niikura; (III) Provision of study materials or patients: M \\ Terao; (IV) Collection and assembly of data: M Terao; (V) Data analysis and interpretation: M Terao; (VI) Manuscript writing: All authors; \\ (VII) Final approval of manuscript: All authors. \\ Correspondence to: Dr. Naoki Niikura. Department of Breast and Endocrine Surgery, Tokai University School of Medicine, 143 Shimokasuya, Isehara, \\ Kanagawa 259-1193, Japan. Email: niikura@is.icc.u-tokai.ac.jp.
}

\begin{abstract}
Metastatic breast cancer (MBC) is generally considered incurable. However, MBC which has limited number and sites of metastasis: oligometastatic breast cancer (OMBC) may have definitive remission with individualized, metastasis-directed ablative therapies and multidisciplinary management, such as surgery and radiotherapy. Earlier detection of primary metastatic breast cancer using accurate imaging technologies may allow the initiation of therapy for OMBC. In breast cancer, the lymph nodes, liver, bone, lung, and brain are common sites of metastasis. Additional studies are recommended if signs or symptoms indicate metastasis. The imaging modality should be selected for suspicious locations or original metastatic regions. The standard staging studies are ultrasonography for lymph node metastasis, abdominal and/or pelvic contrast-enhanced CT for liver metastasis, chest CT for pulmonary metastasis, and bone scan for bone metastasis. FDG-PET/CT may be helpful for bone or liver metastasis. MRI may be considered for liver, soft tissue, or brain metastasis.
\end{abstract}

Keywords: Breast cancer; oligometastasis; diagnosis; imaging

Submitted Dec 13, 2019. Accepted for publication Jan 02, 2020.

doi: $10.21037 /$ tcr.2020.01.04

View this article at: http://dx.doi.org/10.21037/tcr.2020.01.04

\section{Introduction}

Metastatic breast cancer (MBC) is generally considered incurable. However, MBC which has limited number and limited sites of metastasis: oligometastatic breast cancer $(\mathrm{OMBC})$ may have definitive remission with individualized, metastasis-directed ablative therapies and multidisciplinary management, such as surgery and radiotherapy. Earlier detection of primary metastatic breast cancer using accurate imaging technologies may allow the initiation of therapy for OMBC before cancer cells spread widely. Recently, OMBCs have been increasingly detected due to improved imaging and diagnostics. Many clinical trials are investigating the benefit of these local therapeutic approaches across several cancer sites. Patients are required to be correctly included and followed with appropriate imaging (1).

Imaging requirements in recognizing $\mathrm{OMBC}$ depend on metastatic location and timing of measurement in relation to their treatment (1). In breast cancer, OMBC accounts for $20 \%$ of all MBCs $(2,3)$ and has resulted in significantly longer survival in the last few years (4). It is still controversial whether long-term survival in this subset is due to the selection of patients whose tumors have indolent disease biology or effects of therapy (4). Moreover, we need to select these patients with curable OMBCs that have limited number and have limited sites of metastasis from patients with incurable MBC. There are some imaging guidelines and recommendations for patients to be included in treatment for oligometastasis by primary disease site, metastasis location, and timing in the disease cycle (1).

\section{Diagnosis of the point in disease cycle}

The most common setting of the needs for the diagnosis of oligometastasis would be at the timing of diagnosis of primary breast cancer. 
In the diagnosis of primary breast cancer, patients with node-positive breast cancer may have metastasis. In breast cancer, the lymph nodes, liver, bone, lung, and brain are common sites of metastasis. The National Comprehensive Cancer Network (NCCN) guidelines (version 3; 2019) recommend for additional studies only if directed by signs or symptoms for clinical stages T0-3, N1, M0 or T1-3, N0, M0 as workup. If there are elevated alkaline phosphatase levels, abnormal liver function tests, abdominal symptoms, or abnormal physical examination (PE) results in the abdomen or pelvis, abdominal and/or pelvic contrastenhanced diagnostic computed tomography (CT) is recommended. If pulmonary symptoms are present, chest contrast-enhanced diagnostic CT will also be considered. Bone scan (BS) is indicated if there is localized bone pain or elevated alkaline phosphatase level.

Positron emission tomography (PET) using ${ }^{18} \mathrm{~F}$-fluorodeoxyglucose (FDG) may be helpful in situations where standard staging studies are equivocal or suspicious for bone or liver metastases. MRI may be considered for suspicions of liver, soft tissue, or brain metastases.

Occasionally, we recognize the clinical complete response (cCR) in the imaging of the effect measurement after MBC treatment. We may be able to aim to cure of MBC with no findings of metastasis or the findings of oligometastasis alone on the image with additional local therapy as SRT and/or surgery. The imaging modality should be selected for suspicious locations or original metastatic regions as described above.

\section{Locations of MBC}

\section{Lymph node metastasis}

Lymph nodes are the first regional site of metastasis in many cancer types, including breast cancer (5). In the primary diagnosis, axillary lymph nodes are assessed by ultrasonography (US) or other imaging studies, as necessary, and percutaneous fine needle aspiration or core needle biopsy of suspicious lymph nodes. US is inexpensive, ubiquitously available, and the method of choice for lymph node biopsy guidance. Breast lymphatic drainage generally starts from level I to III, and finally into the thorax (6). Metastases generally occur after a tumor has spread to the axillary lymph nodes. Metastases in the ipsilateral infraclavicular (N3a), internal mammary and axillary (N3b), and supraclavicular (N3c) lymph nodes are considered inoperable as locally advanced breast cancer (the American Joint Committee on Cancer staging system). We should confirm the diagnosis of $\mathrm{N} 3$ in selecting the methods of treatment to distinguish operable or inoperable lymph nodes. Even if the diagnosis of nodal staging N3 is confirmed, without other metastases or with a small number of metastatic tumors in one or two other parts of the body, it may be considered curable oligometastasis.

The advantages and disadvantages of lymph node imaging in primary breast cancer were reviewed (5). PE, mammography (MG), and US are commonly performed in the primary examination. In nodal diagnosis, $\mathrm{PE}$ has low sensitivity (SE) (30\%) [specificity (SP), 94\%], and MG has moderate SE (66.9\%) (SP, 80.8\%). In axillary lymph nodes, US has higher SE and is useful for fine needle aspiration.

FDG-PET allows the identification of increased glucose metabolism, which is common in malignant tumors. In identifying advanced axillary disease and metastatic nodal spread outside the axillary lymph nodes, PET combined with CT (PET/CT) reportedly had SE of $64 \%$ and SP of 93\% (pooled estimate, SE, 59-63\%; SP, 90-95\%) (5). PET/ CT may be useful in identifying advanced axillary diseases and unexpected metastatic nodal spread outside the axillary lymph nodes, such as infraclavicular and supraclavicular and contralateral axillary lymph nodes, in patients with large primary tumors $(>3 \mathrm{~cm})$ who underwent other examinations, such as chest $\mathrm{CT}$, liver US, and $\mathrm{BS}$, to rule out distant metastasis (7). Nevertheless, PET/CT is not yet sufficiently sensitive in the detection of primary breast cancer or evaluation of axillary lymph nodes in early-stage breast cancer (stages I and II). Locally advanced breast cancer with a large tumor (T3 or greater) or advanced axillary disease (N2) without evidence of distant metastasis may be appropriate for PET/CT.

\section{Bone metastasis}

The skeleton is the most common site of metastasis in breast cancer. It is affected in approximately $50-70 \%$ of patients with relapse and is the sole site of disease in $28-44 \%(8,9)$. It is important that the site of relapse is localized in the skeleton to diagnose oligometastasis (10). Imaging has always played an important role in the diagnosis of bone metastasis in breast cancer, and planar ${ }^{99}$ Tc-diphosphonate BS remains widely used. CT and MRI are the common whole-body (WB) morphological imaging modalities. However, they are less sensitive in bone disease, which relies on cortical and trabecular destruction. Therefore, in bone metastases, CT is supplemented with 
BS with its high SE but poor SP (10). The first choice for screening should be BS. Because this method shows bone mineral turnover and provides only direct information about tumor activity, it is less sensitive in detecting lytic and/ or indolent metastatic lesions compared to PET/CT. FDGPET shows increased metabolic activity in tumor cells and can detect both osteoblastic and osteolytic lesions at an earlier stage. It may play a complementary role in detecting bone metastasis with bone scintigraphy in patients with breast cancer with suspected bone metastasis and in bone tumor response assessment (11-13). The diagnostic accuracies of PET/CT may be superior to those of BS in patients with suspected bone metastases from breast cancer (12). If patients have any indication of bone metastasis, such as bone pain, elevated tumor marker level, abnormal radiology results, increased alkaline phosphatase level, and locally advanced breast cancer despite negative BS findings, PET/CT will be helpful in the detection of bone oligometastasis.

\section{Lung metastasis}

The incidence of lung metastasis can reach up to $40 \%$ in triple-negative breast cancer (TNBC) compared with only $20 \%$ in non-TNBC (14). The survival records of patients with lung metastasis revealed that hormone receptorpositive breast cancers had the best clinical outcome, while HER2+ cancers and TNBC had the worst prognoses (15). Relying on coregistered anatomical and metabolic PET/CT images, many inflammatory lesions and physiological FDG uptake can be more easily confirmed as negative, while small malignant lesions presenting with low FDG uptake, such as subcentimeter lung nodules or small sclerotic bone lesions, can be well characterized in CT (16-18). In one of the largest FDG-PET/CT retrospective studies, in patients with breast cancer suspected of recurrent metastasis who were followed for at least 6 months, the SE of FDG-PET in detecting metastases was $98.7 \%$, but the SE in detecting metastatic lesions in the brain and lungs was slightly lower compared to that in conventional imaging $(18,19)$. In the diagnosis of primary hormone receptor-positive breast cancer, patients with node-positive breast cancer with high risk of distant metastasis may be recommended to undergo chest CT.

\section{Liver metastasis}

Breast cancer with liver metastasis has a poor outcome if left untreated, with a survival period ranging from 4 to
8 months (20). However, if recurrences or distant metastases may be isolated to a few lesions with limited numbers, individualized, metastasis-directed ablative therapies, including surgery, transcatheter arterial chemoembolization, radiofrequency ablation, and stereotactic body radiotherapy, with systemic chemotherapeutic agents may improve outcomes. MRI is superior to US, CT and FDG-PET/CT in the correct depiction and characterization of liver lesions $(21,22)$. The SE of FDG-PET/CT is limited to detect small liver metastasis $(<1 \mathrm{~cm}$ diameter) because of liver motion and poor spatial resolution. Whole body imaging, such as WB-MRI or FDG-PET/CT, has the advantage of multiorgan evaluation (1). In a study on 51 patients, 30 of whom had a total of 282 distant metastases, PET/MRI offered better SE in liver and possibly bone metastases, while PET/CT remained the best imaging modality for pulmonary metastases (23). Moreover, FDG-PET/MRI classified tumor lesions as malignant or benign lesions better than FDG-PET/CT, an important consideration in recurrent disease (24). If the primary clinical aim is to detect or exclude liver metastases, MRI with liver-specific contrast agents may be helpful, especially in assessing small lesions. Furthermore, if WB-MRI or PET/MRI is available, it may be helpful (1).

\section{Brain metastasis}

An estimated $10-30 \%$ of patients with breast cancer will eventually have brain metastases (25). Breast cancer is the second most common cause of brain metastasis, after lung cancer. Brain metastasis usually occurs late in the clinical history of breast cancer and often in the presence of extracranial metastasis (26). According to a recent Austrian study, the central nervous system is the sole site of metastatic disease in approximately $17 \%$ of patients (27). In patients with early-stage breast cancer, the cumulative incidence rates of brain metastasis are highest in those with HER2 + cancers and TNBCs and lowest in ER-positive disease $(28,29)$. Current oncological guidelines recommend that brain imaging should not be routinely performed in asymptomatic patients (European Society of Medical Oncology). When patients have suspicious neurological symptoms, brain contrast-enhanced MRI may be helpful in detecting brain metastases (NCCN). No data showing that effective screening prolongs overall survival and prevents serious symptoms are available; therefore, the detection of early brain involvement due to metastasis relies on aggressive monitoring of relevant symptoms (30). However, 
early confirmation of brain metastasis as oligometastasis is critical in conducting an intervention and minimizing irreversible damage to the nervous system. When patients with breast cancer have some neurological symptoms, especially in HER2 + cancer or TNBC, brain contrastenhanced MRI may be recommended.

\section{Breast cancer subtypes and sites of distant metastasis}

The pathological subtypes of breast cancer have different metastatic behaviors with respect to the sites of distant metastasis (31).

This knowledge helps us to determine the appropriate strategy for screening and follow-up. The incidence of bone metastasis in $\mathrm{HR}+/ \mathrm{HER} 2+$ subtype was up to $5.1 \%$. Further, patients with $\mathrm{HR}-/ \mathrm{HER} 2$ + subtype had a higher risk of brain metastasis $(\mathrm{OR}=1.978)$ compared to those with $\mathrm{HR}+$ / HER2 - subtype. Additionally, liver metastasis was more frequently observed in the HER2 + subtypes compared with HER2 - subtypes. Patients with TNBC primarily presented with lung metastasis, but the risk of lung metastasis was not significantly different in all subtypes.

\section{Limitations}

There are some risks of false positive findings, and these results cause overdiagnosis and unnecessary treatment. An Australian study showed that staging CT of the abdomen and pelvis is associated with high incidence of false positive rates, $15 \%$, especially in early breast cancer and asymptomatic synchronous metastasis (32). MRI has a high diagnostic accuracy in the evaluation of hepatic lesions but has a risk for false positive results (33). The maximum standardized uptake value in FDG-PET is used to assess the extent of radiotracer uptake, with the cutoff value of 2.0 commonly used to differentiate benign and malignant tumors (34). However, the ability of PET to differentiate malignant tumors from benign lesions is limited (35-37). Several studies have reported false positive of benign lesions, including hibernoma, fibrous dysplasia, neurofibroma, schwannoma, desmoids, and sarcoidosis, as malignant by PET based on high radiotracer uptake $(36,38,39)$. They reported that the false-positive rate of malignancy, increased ${ }^{18} \mathrm{~F}$-FDG uptake in benign lesions, was up to $15-25 \%$ $(39,40)$. There are limitations in the accuracy of diagnostic tools in the detection and assessment of malignant lesions. It shows the importance of a complete workup of suspected metastasis in patients with a primary or previous history of malignancy.

\section{Conclusions}

Recently, the incidence of oligometastasis has increased based on improved imaging and diagnostic modalities, but these techniques are insufficient. The false positive results by imaging cause overdiagnosis and unnecessary treatment. Patients who were diagnosed with stage IV may lose the chance of undergoing standard therapy to treat primary breast cancer. A workup for oligometastasis should include needle or open biopsy to confirm metastasis and rule out benign lesions or other malignancies.

\section{Acknowledgments}

Funding: None.

\section{Footnote}

Provenance and Peer Review: This article was commissioned by the Guest Editors (Tadahiko Shien and Kaori Terata) for the series "Loco regional therapy for metastatic breast cancer" published in Translational Cancer Research. The article was sent for external peer review organized by the Guest Editors and the editorial office.

Conflicts of Interest: The series "Loco-regional therapy for metastatic breast cancer" was commissioned by the editorial office without any funding or sponsorship. N Niikura has received research funding to Tokai University from Novartis, Bristol-Myers Squibb, Chugai Pharmaceutical Co, Nihon Medi-Physics Co. Ltd. MSD and Daiichi-Sankyo, honoraria, consultancy, and speaker fees from AstraZeneca, Novartis, Eisai and Pfizer, outside the submitted work. The other authors declare no competing interests.

Ethical Statement: The authors are accountable for all aspects of the work in ensuring that questions related to the accuracy or integrity of any part of the work are appropriately investigated and resolved.

Open Access Statement: This is an Open Access article distributed in accordance with the Creative Commons Attribution-NonCommercial-NoDerivs 4.0 International License (CC BY-NC-ND 4.0), which permits the noncommercial replication and distribution of the article with 
the strict proviso that no changes or edits are made and the original work is properly cited (including links to both the formal publication through the relevant DOI and the license). See: https://creativecommons.org/licenses/by-nc-nd/4.0/.

\section{References}

1. deSouza NM, Liu Y, Chiti A, et al. Strategies and technical challenges for imaging oligometastatic disease: recommendations from the European Organisation for Research and Treatment of Cancer imaging group. Eur J Cancer 2018;91:153-63.

2. Méry B, Moriceau G, Vallard A, et al. Oligometastatic breast cancer: therapeutic implications of a new paradigm. Rev Med Brux 2018;39:111-3.

3. Di Lascio S, Pagani O. Oligometastatic breast cancer: a shift from palliative to potentially curative treatment? Breast Care (Basel) 2014;9:7-14.

4. Rastogi S, Gulia S, Bajpai J, et al. Oligometastatic breast cancer: a mini review. Indian J Med Paediatr Oncol 2014;35:203-6.

5. Marino MA, Avendano D, Zapata P, et al. Lymph node imaging in patients with primary breast cancer: concurrent diagnostic tools. Oncologist 2019;25:e231-e242.

6. Manca G, Volterrani D, Mazzarri S, et al. Sentinel lymph node mapping in breast cancer: a critical reappraisal of the internal mammary chain issue. Q J Nucl Med Mol Imaging 2014;58:114-26.

7. Robertson IJ, Hand F, Kell MR. FDG-PET/CT in the staging of local/regional metastases in breast cancer. Breast 2011;20:491-4.

8. Plunkett TA, Smith P, Rubens RD. Risk of complications from bone metastases in breast cancer. implications for management. Eur J Cancer 2000;36:476-82.

9. Wei S, Li Y, Siegal GP, et al. Breast carcinomas with isolated bone metastases have different hormone receptor expression profiles than those with metastases to other sites or multiple organs. Ann Diagn Pathol 2011;15:79-83.

10. Cook GJ, Azad GK, Goh V. Imaging bone metastases in breast cancer: staging and response assessment. J Nucl Med 2016;57 Suppl 1:27S-33S.

11. Abe K, Sasaki M, Kuwabara Y, et al. Comparison of 18FDG-PET with 99mTc-HMDP scintigraphy for the detection of bone metastases in patients with breast cancer. Ann Nucl Med 2005;19:573-9.

12. Niikura N, Hashimoto J, Kazama T, et al. Diagnostic performance of (18)F-fluorodeoxyglucose PET/CT and bone scintigraphy in breast cancer patients with suspected bone metastasis. Breast Cancer 2016;23:662-7.

13. Hayashi N, Costelloe CM, Hamaoka T, et al. A prospective study of bone tumor response assessment in metastatic breast cancer. Clin Breast Cancer 2013;13:24-30.

14. Foulkes WD, Smith IE, Reis-Filho JS. Triple-negative breast cancer. N Engl J Med 2010;363:1938-48.

15. Yhim HY, Han SW, Oh DY, et al. Prognostic factors for recurrent breast cancer patients with an isolated, limited number of lung metastases and implications for pulmonary metastasectomy. Cancer 2010;116:2890-901.

16. Veit-Haibach P, Antoch G, Beyer T, et al. FDG-PET/ $\mathrm{CT}$ in restaging of patients with recurrent breast cancer: possible impact on staging and therapy. Br J Radiol 2007;80:508-15.

17. Tatsumi M, Cohade C, Mourtzikos KA, et al. Initial experience with FDG-PET/CT in the evaluation of breast cancer. Eur J Nucl Med Mol Imaging 2006;33:254-62.

18. Gaeta CM, Vercher-Conejero JL, Sher AC, et al. Recurrent and metastatic breast cancer PET, PET/CT, PET/MRI: FDG and new biomarkers. Q J Nucl Med Mol Imaging 2013;57:352-66.

19. Manohar K, Mittal BR, Senthil R, Kashyap R, Bhattacharya A, Singh G. Clinical utility of F-18 FDG $\mathrm{PET} / \mathrm{CT}$ in recurrent breast carcinoma. Nucl Med Commun 2012;33:591-6.

20. Adam R, Aloia T, Krissat J, et al. Is liver resection justified for patients with hepatic metastases from breast cancer? Ann Surg 2006;244:897-907; discussion -8.

21. Antoch G, Vogt FM, Freudenberg LS, et al. Whole-body dual-modality PET/CT and whole-body MRI for tumor staging in oncology. JAMA 2003;290:3199-206.

22. Heusch P, Nensa F, Schaarschmidt B, et al. Diagnostic accuracy of whole-body PET/MRI and whole-body PET/ CT for TNM staging in oncology. Eur J Nucl Med Mol Imaging 2015;42:42-8.

23. Melsaether AN, Raad RA, Pujara AC, et al. Comparison of whole-body (18)F FDG PET/MR imaging and wholebody (18)F FDG PET/CT in terms of lesion detection and radiation dose in patients with breast cancer. Radiology 2016;281:193-202.

24. Sawicki LM, Grueneisen J, Schaarschmidt BM, et al. Evaluation of (1)(8)F-FDG PET/MRI, (1)(8)F-FDG PET/ CT, MRI, and CT in whole-body staging of recurrent breast cancer. Eur J Radiol 2016;85:459-65.

25. Lin NU, Bellon JR, Winer EP. CNS metastases in breast cancer. J Clin Oncol 2004;22:3608-17.

26. Arslan C, Dizdar O, Altundag K. Chemotherapy and biological treatment options in breast cancer patients with 
brain metastasis: an update. Expert Opin Pharmacother 2014;15:1643-58.

27. Berghoff AS, Bago-Horvath Z, Ilhan-Mutlu A, et al. Brain-only metastatic breast cancer is a distinct clinical entity characterised by favourable median overall survival time and a high rate of long-term survivors. Br J Cancer 2012;107:1454-8.

28. Kennecke H, Yerushalmi R, Woods R, et al. Metastatic behavior of breast cancer subtypes. J Clin Oncol 2010;28:3271-7.

29. Arvold ND, Oh KS, Niemierko A, et al. Brain metastases after breast-conserving therapy and systemic therapy: incidence and characteristics by biologic subtype. Breast Cancer Res Treat 2012;136:153-60.

30. Niikura N, Saji S, Tokuda Y, et al. Brain metastases in breast cancer. Jpn J Clin Oncol 2014;44:1133-40.

31. Wu Q, Li J, Zhu S, et al. Breast cancer subtypes predict the preferential site of distant metastases: a SEER based study. Oncotarget 2017;8:27990-6.

32. James J, Teo M, Ramachandran V, et al. Performance of CT scan of abdomen and pelvis in detecting asymptomatic synchronous metastasis in breast cancer. Int J Surg 2017;46:164-9.

33. Noone TC, Semelka RC, Balci NC, et al. Common occurrence of benign liver lesions in patients with newly diagnosed breast cancer investigated by MRI for suspected liver metastases. J Magn Reson Imaging 1999;10:165-9.

Cite this article as: Terao M, Niikura N. Diagnosis of oligometastasis. Transl Cancer Res 2020;9(8):5032-5037. doi: 10.21037/tcr.2020.01.04
34. Griffeth LK, Dehdashti F, McGuire AH, et al. PET evaluation of soft-tissue masses with fluorine-18 fluoro-2deoxy-D-glucose. Radiology 1992;182:185-94.

35. Aoki J, Watanabe H, Shinozaki T, et al. FDG-PET for preoperative differential diagnosis between benign and malignant soft tissue masses. Skeletal Radiol 2003;32:133-8.

36. Tian R, Su M, Tian Y, et al. Dual-time point PET/CT with F-18 FDG for the differentiation of malignant and benign bone lesions. Skeletal Radiol 2009;38:451-8.

37. Lindskog DM, Nikkhou K, Talusan P. False-positive positron emission tomography in patients with history of malignancy. J Clin Oncol 2011;29:e582-5.

38. Metser U, Even-Sapir E. Increased (18) F-fluorodeoxyglucose uptake in benign, nonphysiologic lesions found on whole-body positron emission tomography/computed tomography (PET/CT): accumulated data from four years of experience with PET/ CT. Semin Nucl Med 2007;37:206-22.

39. Schulte M, Brecht-Krauss D, Heymer B, et al. Grading of tumors and tumorlike lesions of bone: evaluation by FDG PET. J Nucl Med 2000;41:1695-701.

40. Metser U, Miller E, Lerman H, et al. Benign nonphysiologic lesions with increased 18F-FDG uptake on PET/CT: characterization and incidence. AJR Am J Roentgenol 2007;189:1203-10. 